\title{
Uranium isotopes in Permian marine carbonates and calcitic brachiopods: validation of the global paleoredox proxy and implications for ocean oxygenation in the Permian
}

WENQIAN WANG ${ }^{1}$, FEIFEI ZHANG ${ }^{1,2 *}$, SHUZHONG SHEN $^{1 *}$, MARTIN BIZZARRO ${ }^{2}$, ClAAUDIO GARBELLI ${ }^{3}$, TAIS W. DAHL ${ }^{2}$

${ }^{1}$ School of Earth Sciences and Engineering, Nanjing University, Nanjing, 210023, China (*correspondence: fzhang@nju.edu.cn; szshen@nju.edu.cn)

${ }^{2}$ GLOBE Institute, University of Copenhagen, 1350

Copenhagen, Denmark

3 Nanjing Institute of Geology and Palaeontology, Chinese Academy of Sciences, Nanjing, 210008, China

Uranium isotopes $\left(\delta^{238} \mathrm{U}\right)$ in marine carbonate sediments have widely been used to place quantitative constraints on the oxygenation state of the oceans in geological history. However, marine carbonate sediments precipate partly during diagenesis, and suffer from post-depositional alteration processes, imposing a positive, albeit variable, $\delta^{238} \mathrm{U}$ offset in the sediments relative to contemporaneous seawater. In contrast, calcitic brachiopod shells reliably record the $\delta^{238} \mathrm{U}$ value of the seawater in which they grew (1), and they consist of a mineral more resistant to diagenetic and postdepositional alteration (2). The low U content in brachiopod shells, however, pose a challenge to precise $\delta^{238} U$ analysis. Using a refined chemical purification protocol and employing $10^{13} \Omega$ resistors on MC-ICPMS Neptune Plus, we present 47 $\delta^{238} \mathrm{U}$ measurements from 21 brachiopod shells and their host carbonate rocks to test the robustness of brachiopods for recording the original $\delta^{238} \mathrm{U}$ composition of Permian seawater and the secular variation in the global ocean oxygenation states.

We used SEM, CL microscopy and the elemental analyses to determine the preservation of the shells. We find that $54 \%, 27 \%$ and $19 \%$ of the shells have lower, same and higher $\delta^{238} \mathrm{U}$ values than their host rocks, respectively, demonstrating overall better preservation of $\delta^{238} U$ in brachiopod shells. Using the $\delta^{238} \mathrm{U}$ records of best preserved brachiopod shells, we infer widespread oceanic anoxia in the earliest Permian oceans, a temporal recovery to more oxygenated ocean conditions during the Kungurian, and a return to anoxic conditions in the late Guadalupian to Lopingian oceans.

[1] Livermore et al., (in review). [2] Del Rey et al. (in review) 